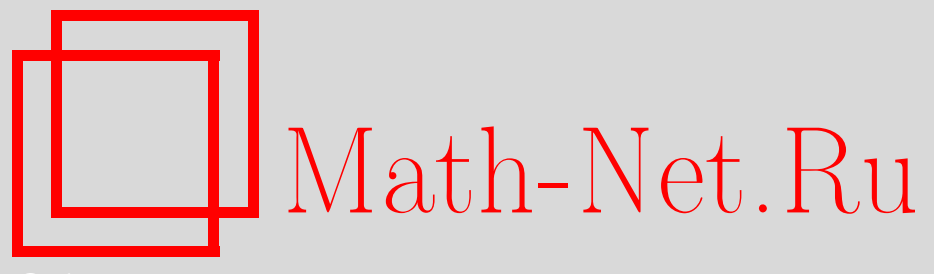

В. Н. Соболев, Об асимптотических разложениях в центральной предельной теореме, Теория вероятн. и ее примен., 2007, том 52, выпуск 3, 490-505

DOI: https://doi.org/10.4213/tvp75

Использование Общероссийского математического портала Math-Net.Ru подразумевает, что вы прочитали и согласны с пользовательским соглашением

http: //www . mathnet.ru/rus/agreement

Параметры загрузки:

IP : 3.81 .55 .215

26 апреля 2023 г., $11: 58: 17$

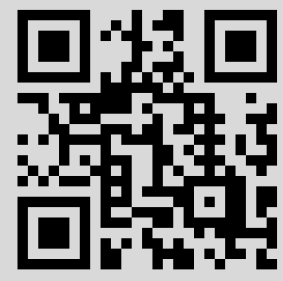




\section{ОБ АСИМПТОТИЧЕСКИХ РАЗЛОЖЕНИЯХ В ЦЕНТРАЛЬНОЙ ПРЕДЕЛЬНОЙ ТЕОРЕМЕ}

В статье приводятся новые явные оценки точности для асимптотических разложений в центральной предельной теореме.

Ключевие слова и фразы: центральная предельная теорема, асимптотические разложения, точность разложения.

Введение. Пусть $X_{1}, X_{2}, \ldots$ - независимые одинаково распределенные случайные величины с нулевым средним и единичной дисперсией. Обозначим $P$ распределение $X_{1}, P_{n}$ - распределение нормированной суммы $\left(X_{1}+\cdots+X_{n}\right) n^{-1 / 2}$, а через $\Phi-$ стандартный нормальный закон с плотностью $\varphi(x)=(\sqrt{2 \pi})^{-1 / 2} e^{-x^{2} / 2}$. Мы предполагаем, что все используемые в дальнейшем моменты $\mathbf{E} X_{1}^{m}$ и $\mathbf{E}\left|X_{1}\right|^{m}$ существуют. Далее мы будем использовать величины $\alpha_{m}=\mathbf{E} X_{1}^{m} / m !, \beta_{m}=\mathbf{E}\left|X_{1}\right|^{m} / m !$. Из наших предположений следует, что $\alpha_{0}=1, \alpha_{1}=0, \alpha_{2}=\frac{1}{2}$. Соответствующие величины для стандартного нормального закона обозначим $\alpha_{m}(\varphi), \beta_{m}(\varphi)$. Так, $\alpha_{2 j}(\varphi)=1 /\left(2^{j} j !\right)$ для $j=0,1,2, \ldots$

Мы также будем использовать моменты Чебышёва-Эрмита $\theta_{k}(P)$ (далее вместо $\theta_{k}(P)$ будем часто писать $\theta_{k}$ ), которые определяются формулой $\theta_{k}(P)=(1 / k !) \int_{-\infty}^{\infty} H_{k}(x) P(d x)$, где $H_{k}(x)$ - многочлены Чебышёва-Эрмита. В разных источниках многочлены Чебышёва-Эрмита определяются по-разному. Мы будем называть многочленами Чебышёва-Эрмита многочлены, которые определяются формулой $H_{k}(x)=$ $(-1)^{k} \varphi^{(k)}(x) / \varphi(x)$. Для $H_{k}(x)$ и $\theta_{k}$ хорошо известны равенства

$$
H_{k}(x)=k ! \sum_{j=0}^{[k / 2]}(-1)^{j} \alpha_{2 j}(\varphi) \frac{x^{k-2 j}}{(k-2 j) !} \quad \text { и } \quad \theta_{k}=\sum_{j=0}^{[k / 2]}(-1)^{j} \alpha_{2 j}(\varphi) \alpha_{k-2 j} .
$$

Нам также потребуются числа

$$
\left\|\theta_{k}\right\|=\beta_{k}+\left\|\theta_{k}^{(k-2)}\right\|, \quad \text { где } \quad\left\|\theta_{k}^{(k-2)}\right\|=\sum_{j=1}^{[k / 2]}\left|\alpha_{k-2 j}\right| \alpha_{2 j}(\varphi) .
$$

* Российский государственный торгово-экономический университет, ул. Смольная, 36, 125195 Москва, Россия; e-mail: 6li@mail.ru; sobolev_vn@mail.ru 
Обозначим через $f(t)$ характеристическую функцию распределения $P$. Далее будем предполагать, что для некоторого неотрицательного $\nu$ функция $|f(t)|^{\nu}$ интегрируема на всей числовой оси. Обозначим

$$
\alpha(T)=\max \{|f(t)|: t \geqslant T\}, \quad A_{n}(T)=\frac{\sqrt{n}}{\pi} \alpha^{n-\nu}(T) \int_{T}^{\infty}|f(t)|^{\nu} d t .
$$

Заметим, что выполнение условия $\int_{-\infty}^{\infty}|f(t)|^{\nu} d t<\infty$ гарантирует существование непрерывной плотности $p_{n}(x)$ у распределения $P_{n}$ при всех $n \geqslant \nu$, эту плотность можно вычислять при помощи формулы обращения для преобразования Фурье:

$$
p_{n}(x)=(2 \pi)^{-1} \int_{-\infty}^{\infty} e^{-i t x} f^{n}\left(\frac{t}{\sqrt{n}}\right) d t
$$

кроме того, величина $\alpha(T)$ строго меньше единицы для любого $T>0$.

Для получения разложений для $p_{n}(x)$ мы будем использовать вспомогательные заряды $Q$ с характеристическими функциями $g(t)$. Явный вид $g(t)$ будет приведен ниже. Обозначим $q_{n}(x)$ плотность $n$-кратной нормированной свертки $Q_{n}$ заряда $Q$, характеристическая функция заряда $Q_{n}$ равна $g^{n}(t / \sqrt{n})$. Плотность $q_{n}(x)$ при любом $n \geqslant 1$ можно вычислять по формуле обращения для преобразования Фурье:

$$
q_{n}(x)=\frac{1}{2 \pi} \int_{-\infty}^{\infty} e^{-i t x} g^{n}\left(\frac{t}{\sqrt{n}}\right) d t .
$$

Нам понадобятся неотрицательные четные функции $\mu(t) \geqslant e^{-t^{2} / 2}$ и числа $0<T \leqslant 1$ такие, что $|f(t)| \leqslant \mu(t)$ при $|t| \leqslant T$. Для пары $(\mu, T)$ определим величины

$$
\begin{gathered}
B_{l, n-k}=\frac{1}{2 \pi} \int_{-T \sqrt{n}}^{T \sqrt{n}}|t|^{l} \mu^{n-k}\left(\frac{t}{\sqrt{n}}\right) d t, \quad B_{l}=\frac{1}{2 \pi} \int_{-\infty}^{\infty}|t|^{l} e^{-t^{2} / 2} d t, \\
L_{l}(u)=\frac{1}{2 \pi} \int_{|t| \geqslant u}|t|^{l} e^{-t^{2} / 2} d t .
\end{gathered}
$$

При достаточно широких условиях на распределение $P$ пару $(\mu, T)$ можно подобрать так, чтобы величины $B_{l, n-k}$ при росте $n$ стремились к величинам $B_{l}[1]$.

Сформулируем условие, которое будет нами часто использоваться.

У с л о в и е 1 . У распределения $P$ с нулевым средним и единичной дисперсией существует конечный абсолютный момент порядка $m+2$; существует число $\nu>0$ такое, что для характеристической функции $f(t)$ распределения $P$ выполняется условие $\int_{-\infty}^{\infty}|f(t)|^{\nu} d t<\infty$; неотрицательная четная функция $\mu(t)$ и число $0<T \leqslant 1$ таковы, что $|f(t)| \leqslant \mu(t)$ при $|t| \leqslant T$. 
1. Результаты. Мы получим асимптотические разложения в центральной предельной теореме (ЦПТ) для независимых одинаково распределенных случайных величин $X_{1}, X_{2}, \ldots$, у которых конечны моменты $\beta_{m+2}, m=4,5,6$. Эти разложения занимают промежуточное место между разложениями Эджворта-Крамера и короткими разложениями Грама-Шарлье [1].

Утверждение теоремы 1 отличается от соответствующего результата, приведенного в [1], главным образом тем, что в теореме 1 нет ограничения $\theta_{4}(P) \leqslant 6$, присутствующего в [1]. Аналога теоремы 3 в [1] нет.

Поскольку отличия в доказательствах теорем 1-3 носят технический, а не идейный характер, мы дадим полное доказательство лишь для теоремы 1, а затем укажем, как следует модифицировать это доказательство, с тем чтобы получить утверждения теорем 2 и 3 . Здесь следует заметить, что громоздкость формулировок теорем связана не с техническими проблемами, а с существом дела.

Теорема 1. Пусть справедливо условие 1 при $m=4$. Тогда для любьх $x$ и $n \geqslant \max (\nu, 6)$

$$
\begin{aligned}
p_{n}(x)= & \varphi(x)+\frac{\theta_{3}}{\sqrt{n}} H_{3}(x) \varphi(x)+\frac{\theta_{3}^{2}}{2(\sqrt{n})^{2}} H_{6}(x) \varphi(x)+\frac{\theta_{4}}{(\sqrt{n})^{2}} H_{4}(x) \varphi(x) \\
& +\frac{\theta_{3}^{3}}{6(\sqrt{n})^{3}} H_{9}(x) \varphi(x)+\frac{\theta_{5}}{(\sqrt{n})^{3}} H_{5}(x) \varphi(x) \\
& +\frac{n-1}{n} \frac{\theta_{3} \theta_{4}}{(\sqrt{n})^{3}} H_{7}(x) \varphi(x)+R(x),
\end{aligned}
$$

$2 \partial e$

$$
\begin{aligned}
|R(x)| \leqslant & \frac{\left\|\theta_{6}\right\| B_{6, n-1}}{(\sqrt{n})^{4}}+\frac{\theta_{3}^{2}}{2} \frac{B_{6, n}}{(\sqrt{n})^{4}}+\frac{\left|\theta_{4}\right|\left\|\theta_{4}\right\| B_{8, n-1}}{2(\sqrt{n})^{4}}+\frac{\left|\theta_{3} \theta_{5}\right| B_{8}}{(\sqrt{n})^{4}} \\
& +\frac{\theta_{3}^{2}\left|\theta_{4}\right|}{2} \frac{B_{10}}{(\sqrt{n})^{4}}+\frac{\theta_{3}^{4}}{4 !} \frac{B_{12}}{(\sqrt{n})^{4}}+\frac{\left\|\theta_{7}^{(5)}\right\| B_{7, n-1}}{(\sqrt{n})^{5}}+\frac{\left|\theta_{4} \theta_{5}\right| B_{9}}{2(\sqrt{n})^{5}} \\
& +\frac{\left|\theta_{4}\right|\left\|\theta_{5}^{(3)}\right\| B_{9, n-1}}{2(\sqrt{n})^{5}}+\frac{\left|\theta_{3} \theta_{5}\right| B_{8, n}}{(\sqrt{n})^{6}}+\frac{\theta_{3}^{2}\left|\theta_{4}\right| B_{10, n}}{4(\sqrt{n})^{6}}+\frac{\theta_{5}^{2}}{2} \frac{B_{10}}{(\sqrt{n})^{6}} \\
& +\frac{\theta_{5}^{2}}{2} \frac{B_{10, n-1}}{(\sqrt{n})^{8}}+A_{n}(T) \\
& +L_{0}(T \sqrt{n})+\frac{\left|\theta_{4}\right| L_{4}(T \sqrt{n})}{(\sqrt{n})^{2}}+\frac{\left|\theta_{3} \theta_{4}\right| L_{7}(T \sqrt{n})}{(\sqrt{n})^{3}} .
\end{aligned}
$$

Теорема 2. Пусть справедливо условие 1 при $m=5$. Тогда для любых $x$ u $n \geqslant \max (\nu, 7)$

$$
\begin{aligned}
p_{n}(x)= & \varphi(x)+\frac{\theta_{3}}{\sqrt{n}} H_{3}(x) \varphi(x)+\frac{\theta_{3}^{2}}{2(\sqrt{n})^{2}} H_{6}(x) \varphi(x)+\frac{\theta_{4}}{(\sqrt{n})^{2}} H_{4}(x) \varphi(x) \\
& +\frac{\theta_{3}^{3}}{6(\sqrt{n})^{3}} H_{9}(x) \varphi(x)+\frac{\theta_{5}}{(\sqrt{n})^{3}} H_{5}(x) \varphi(x)
\end{aligned}
$$




$$
\begin{aligned}
& +\frac{n-1}{n} \frac{\theta_{3} \theta_{4}}{(\sqrt{n})^{3}} H_{7}(x) \varphi(x)+\frac{\theta_{3}^{4}}{4 !(\sqrt{n})^{4}} H_{12}(x) \varphi(x) \\
& +\left(\frac{n-1}{n}\right)^{2} \frac{\theta_{3}^{2} \theta_{4}}{2(\sqrt{n})^{4}} H_{10}(x) \varphi(x)+\frac{n-1}{n} \frac{\theta_{4}^{2}}{2(\sqrt{n})^{4}} H_{8}(x) \varphi(x) \\
& +\frac{\theta_{3} \theta_{5}}{(\sqrt{n})^{4}} H_{8}(x) \varphi(x)+\left(\theta_{6}-\frac{\theta_{3}^{2}}{2}\right) \frac{1}{(\sqrt{n})^{4}} H_{6}(x) \varphi(x)+R(x),
\end{aligned}
$$

где

$$
\begin{aligned}
|R(x)| \leqslant & \frac{\left\|\theta_{7}\right\| B_{7, n-1}}{(\sqrt{n})^{5}}+\frac{\left|\theta_{4} \theta_{5}\right| B_{9}}{2(\sqrt{n})^{5}}+\left|\theta_{6}-\frac{\theta_{3}^{2}}{2}\right| \frac{\left|\theta_{3}\right| B_{9}}{(\sqrt{n})^{5}}+\left|\theta_{6}-\frac{\theta_{3}^{2}}{2}\right|\left|\theta_{5}\right| \frac{B_{11}}{2(\sqrt{n})^{5}} \\
& +\frac{\left|\theta_{4}\right|\left\|\theta_{5}\right\| B_{9, n-1}}{2(\sqrt{n})^{5}}+\frac{\left|\theta_{3}\right| \theta_{4}^{2} B_{11}}{2(\sqrt{n})^{5}}+\frac{\theta_{3}^{2}\left|\theta_{5}\right| B_{11}}{2(\sqrt{n})^{5}}+\frac{\theta_{3}^{3}\left|\theta_{4}\right|}{2} \frac{B_{13}}{(\sqrt{n})^{5}} \\
& +\frac{\theta_{3}^{5}}{5 !} \frac{B_{15}}{(\sqrt{n})^{5}}+\frac{\left\|\theta_{8}^{(6)}\right\| B_{8, n-1}}{(\sqrt{n})^{6}}+\frac{\left|\theta_{3} \theta_{5}\right| B_{8, n}}{(\sqrt{n})^{6}}+\frac{\theta_{5}^{2}}{2} \frac{B_{10}}{(\sqrt{n})^{6}} \\
& +\frac{\left|\theta_{6}-\theta_{3}^{2} / 2\right|\left\|\theta_{4}\right\| B_{10, n-1}}{2(\sqrt{n})^{6}}+\frac{\left|\theta_{4}\right|\left\|\theta_{6}^{(4)}\right\| B_{10, n-1}}{2(\sqrt{n})^{6}}+\frac{\theta_{3}^{2}\left|\theta_{4}\right| B_{10, n}}{4(\sqrt{n})^{6}} \\
& +\frac{\left\|\theta_{4}\right\| \theta_{4}^{2} B_{12, n-4}}{6(\sqrt{n})^{6}}+A_{n}(T)+\frac{\left\|\theta_{5}^{(3)}\right\| \theta_{4}^{2} B_{13, n-4}}{6(\sqrt{n})^{7}}+\frac{\theta_{4}^{2}\left|\theta_{5}\right| B_{13}}{3 !(\sqrt{n})^{7}} \\
& +\frac{\left|\theta_{3}^{3}\right|}{3 !} \frac{B_{9, n}}{(\sqrt{n})^{7}}+\left|\theta_{6}-\frac{\theta_{3}^{2}}{2}\right|\left\|\theta_{5}^{(3)}\right\| \frac{B_{11, n-1}}{(\sqrt{n})^{7}}+\frac{\theta_{5}^{2}}{2} \frac{B_{10, n-1}}{(\sqrt{n})^{8}} \\
& +\left|\theta_{6}-\frac{\theta_{3}^{2}}{2}\right| \frac{\theta_{3}^{2}}{2} \frac{B_{12, n}}{(\sqrt{n})^{8}}+\frac{\theta_{3}^{2} \theta_{4}^{2} B_{14, n-3}}{12(\sqrt{n})^{8}}+L_{0}(T \sqrt{n})+\frac{\left|\theta_{4}\right| L_{4}(T \sqrt{n})}{(\sqrt{n})^{2}} \\
& +\left|\theta_{6}-\frac{\theta_{3}^{2}}{2}\right| \frac{L_{6}(T \sqrt{n})}{(\sqrt{n})^{4}}+\frac{\left|\theta_{3} \theta_{4}\right| L_{7}(T \sqrt{n})}{(\sqrt{n})^{3}} \\
& +\frac{\theta_{4}^{2} L_{8}(T \sqrt{n})}{2(\sqrt{n})^{4}}+\frac{\left|\theta_{3}^{2} \theta_{4}\right| L_{10}(T \sqrt{n})}{2(\sqrt{n})^{4}}
\end{aligned}
$$

Теорема 3. Пусть справедливо условие 1 при $m=6$. Тогда для любьх $x$ u $n \geqslant \max (\nu, 8)$

$$
\begin{aligned}
p_{n}(x)= & \varphi(x)+\frac{\theta_{3}}{\sqrt{n}} H_{3}(x) \varphi(x)+\frac{\theta_{3}^{2}}{2(\sqrt{n})^{2}} H_{6}(x) \varphi(x)+\frac{\theta_{4}}{(\sqrt{n})^{2}} H_{4}(x) \varphi(x) \\
& +\frac{\theta_{3}^{3}}{6(\sqrt{n})^{3}} H_{9}(x) \varphi(x)+\frac{\theta_{5}}{(\sqrt{n})^{3}} H_{5}(x) \varphi(x) \\
& +\frac{n-1}{n} \frac{\theta_{3} \theta_{4}}{(\sqrt{n})^{3}} H_{7}(x) \varphi(x)+\frac{\theta_{3}^{4}}{4 !(\sqrt{n})^{4}} H_{12}(x) \varphi(x) \\
& +\left(\frac{n-1}{n}\right)^{2} \frac{\theta_{3}^{2} \theta_{4}}{2(\sqrt{n})^{4}} H_{10}(x) \varphi(x)+\frac{n-1}{n} \frac{\theta_{4}^{2}}{2(\sqrt{n})^{4}} H_{8}(x) \varphi(x) \\
& +\frac{\theta_{3} \theta_{5}}{(\sqrt{n})^{4}} H_{8}(x) \varphi(x)+\frac{\left(\theta_{6}-\theta_{3}^{2} / 2\right)}{(\sqrt{n})^{4}} H_{6}(x) \varphi(x)
\end{aligned}
$$




$$
\begin{aligned}
& +\frac{n-1}{n} \frac{\left(\theta_{6}-\theta_{3}^{2} / 2\right) \theta_{3}}{(\sqrt{n})^{5}} H_{9}(x) \varphi(x)+\frac{\theta_{3}^{4}}{4 !(\sqrt{n})^{4}} H_{12}(x) \varphi(x) \\
& +\frac{\theta_{3} \theta_{5}}{(\sqrt{n})^{4}} H_{8}(x) \varphi(x)+\frac{\theta_{7}}{(\sqrt{n})^{5}} H_{7}(x) \varphi(x) \\
& +\frac{n-1}{n} \frac{\theta_{4} \theta_{5}}{(\sqrt{n})^{5}} H_{9}(x) \varphi(x)+\frac{\theta_{3}^{2} \theta_{5}}{2(\sqrt{n})^{5}} H_{11}(x) \varphi(x) \\
& +\frac{C_{n}^{2}}{n^{2}} \frac{\theta_{3} \theta_{4}^{2}}{(\sqrt{n})^{5}} H_{11}(x) \varphi(x)+\left(\frac{n-1}{n}\right)^{3} \frac{\theta_{3}^{3} \theta_{4}}{3 !(\sqrt{n})^{5}} H_{13}(x) \varphi(x) \\
& +\frac{\theta_{3}^{5}}{5 !(\sqrt{n})^{5}} H_{15}(x) \varphi(x)+R(x),
\end{aligned}
$$

где

$$
\begin{aligned}
& |R(x)| \leqslant \frac{\left\|\theta_{8}\right\| B_{8, n-1}}{(\sqrt{n})^{6}}+\frac{\left|\theta_{3} \theta_{5}\right| B_{8, n}}{(\sqrt{n})^{6}}+\frac{\left|\theta_{4}\right|}{2}\left(\left\|\theta_{6}\right\|+\frac{\theta_{3}^{2}}{2}+\left|\theta_{6}-\frac{\theta_{3}^{2}}{2}\right|\right) \frac{B_{10, n-2}}{(\sqrt{n})^{6}} \\
& +\left(\left|\theta_{3} \theta_{7}\right|+\frac{\theta_{5}^{2}}{2}\right) \frac{B_{10}}{(\sqrt{n})^{6}}+\frac{\left\|\theta_{4}\right\| \theta_{4}^{2} B_{12, n-4}}{6(\sqrt{n})^{6}}+\frac{\theta_{3}^{2} \theta_{4}^{2} B_{14}}{4(\sqrt{n})^{6}}+\frac{\theta_{3}^{3}\left|\theta_{5}\right| B_{14}}{3 !(\sqrt{n})^{6}} \\
& +\frac{\theta_{3}^{6}}{6 !} \frac{B_{18}}{(\sqrt{n})^{6}}+\frac{\left|\theta_{3}^{4} \theta_{4}\right|}{4 !} \frac{B_{16}}{(\sqrt{n})^{6}}+\left|\theta_{3} \theta_{4} \theta_{5}\right| \frac{B_{12}}{(\sqrt{n})^{6}}+\frac{\theta_{3}^{3}}{3 !} \frac{B_{9, n}}{(\sqrt{n})^{7}} \\
& +\frac{\left\|\theta_{9}^{(7)}\right\| B_{9, n-1}}{(\sqrt{n})^{7}}+\frac{\left|\theta_{4} \theta_{7}\right| B_{11}}{2(\sqrt{n})^{7}}+\left|\theta_{6}-\frac{\theta_{3}^{2}}{2}\right|\left|\theta_{5}\right| \frac{B_{11}}{(\sqrt{n})^{7}} \\
& +\frac{\left|\theta_{4}\right|}{2} \frac{\left\|\theta_{7}^{(5)}\right\| B_{11, n-2}}{(\sqrt{n})^{7}}+\frac{\theta_{4}^{2}\left|\theta_{5}\right| B_{13, n-2}}{3 !(\sqrt{n})^{7}}+\frac{\left\|\theta_{5}^{(3)}\right\| \theta_{4}^{2} B_{13, n-4}}{6(\sqrt{n})^{7}} \\
& +\frac{\theta_{5}^{2}}{2} \frac{B_{10, n-1}}{(\sqrt{n})^{8}}+\left|\theta_{5} \theta_{7}\right| \frac{B_{12}}{(\sqrt{n})^{8}} \\
& +\frac{1}{2}\left|\theta_{6}-\frac{\theta_{3}^{2}}{2}\right|\left(\left\|\theta_{6}\right\|+\frac{\theta_{3}^{2}}{2}+\frac{\left|\theta_{3} \theta_{4} \theta_{5}\right|}{2}\right) \frac{B_{12, n-2}}{(\sqrt{n})^{8}}+\frac{\theta_{3}^{2} \theta_{4}^{2} B_{14, n-3}}{12(\sqrt{n})^{8}} \\
& +\frac{\left|\theta_{4}\right| \theta_{5}^{2} B_{14}}{2(\sqrt{n})^{8}}+\left|\theta_{6}-\frac{\theta_{3}^{2}}{2}\right|\left|\theta_{7}\right| \frac{B_{13}}{2(\sqrt{n})^{9}}+\left|\theta_{6}-\frac{\theta_{3}^{2}}{2}\right|\left|\theta_{5}\right| \frac{\theta_{3}^{2}}{2} \frac{B_{17}}{(\sqrt{n})^{9}} \\
& +\frac{\theta_{4}^{2}\left|\theta_{5}\right| B_{15}}{3 !(\sqrt{n})^{9}}+\frac{1}{2}\left|\theta_{6}-\frac{\theta_{3}^{2}}{2}\right| \frac{\left\|\theta_{7}^{(5)}\right\| B_{13, n-2}}{(\sqrt{n})^{9}}+\frac{\theta_{7}^{2}}{2} \frac{B_{14}}{(\sqrt{n})^{10}} \\
& +\frac{\left|\theta_{4}\right|}{2} \frac{\theta_{5}^{2}}{2} \frac{B_{14, n-2}}{(\sqrt{n})^{10}}+\frac{1}{2}\left|\theta_{6}-\frac{\theta_{3}^{2}}{2}\right|\left(\frac{\left|\theta_{3} \theta_{5}\right| B_{14, n}}{(\sqrt{n})^{10}}+\frac{\theta_{5}^{2}}{2} \frac{B_{16, n-2}}{(\sqrt{n})^{12}}\right) \\
& +A_{n}(T)+L_{0}(T \sqrt{n})+\left|\theta_{6}-\frac{\theta_{3}^{2}}{2}\right|\left(\frac{L_{6}(T \sqrt{n})}{(\sqrt{n})^{4}}+\left|\theta_{3}\right| \frac{L_{9}(T \sqrt{n})}{(\sqrt{n})^{5}}\right) \\
& +\frac{\theta_{3} \theta_{4}^{2} L_{11}(T \sqrt{n})}{2(\sqrt{n})^{5}}+\frac{\left|\theta_{3}^{3} \theta_{4}\right| L_{13}(T \sqrt{n})}{3 !(\sqrt{n})^{5}}+\frac{\left|\theta_{4}\right| L_{4}(T \sqrt{n})}{(\sqrt{n})^{2}}+\frac{\theta_{4}^{2} L_{8}(T \sqrt{n})}{2(\sqrt{n})^{4}} \\
& +\frac{\left|\theta_{3} \theta_{4}\right| L_{7}(T \sqrt{n})}{(\sqrt{n})^{3}}+\frac{\left|\theta_{4} \theta_{5}\right| L_{9}(T \sqrt{n})}{(\sqrt{n})^{5}}+\frac{\left|\theta_{3}^{2} \theta_{4}\right| L_{10}(T \sqrt{n})}{2(\sqrt{n})^{4}} .
\end{aligned}
$$


2. Доказательство теорем. Для доказательства теорем используются вспомогательные сопровождающие заряды с характеристическими функциями $g_{3}(t)=\exp \left\{(i t)^{2} / 2+\theta_{3}(i t)^{3}\right\}, g_{5}(t)=\exp \left\{(i t)^{2} / 2+\right.$ $\left.\theta_{3}(i t)^{3}+\theta_{5}(i t)^{5}\right\}$ и $g_{7}(t)=\exp \left\{(i t)^{2} / 2+\theta_{3}(i t)^{3}+\theta_{5}(i t)^{5}+\theta_{7}(i t)^{7}\right\}$.

Нам также понадобится лемма 1 из [1], из которой следует, что при $|t|<\sqrt{n}$ и любом натуральном $m \geqslant 2$ для характеристической функции $f(t)$ распределения $P$ с нулевым средним и единичной дисперсией, имеющей конечные моменты до $(m+2)$-го включительно, справедливо равенство

$$
\begin{aligned}
e^{t^{2} /(2 n)} f\left(\frac{t}{\sqrt{n}}\right)= & \sum_{k=0}^{m+1} \theta_{k}\left(\frac{i t}{\sqrt{n}}\right)^{k} \\
& +\gamma e^{t^{2} /(2 n)}\left(\left\|\theta_{m+2}\right\|\left|\frac{t}{\sqrt{n}}\right|^{m+2}+\left\|\theta_{m+3}^{(m+1)}\right\|\left|\frac{t}{\sqrt{n}}\right|^{m+3}\right) .
\end{aligned}
$$

Здесь и далее $\gamma$ обозначает различные величины, для которых справедлива оценка $|\gamma| \leqslant 1$.

Д оказательс т в о т е о ремы 1. При $|t| \leqslant 1$ справедливо тождество

$$
\begin{aligned}
p_{n}(x)-q_{n}(x)= & \frac{1}{2 \pi} \int_{-\infty}^{\infty} e^{-i t x}\left[f^{n}\left(\frac{t}{\sqrt{n}}\right)-g_{5}^{n}\left(\frac{t}{\sqrt{n}}\right)\right] d t \\
= & \frac{1}{2 \pi} \int_{-T \sqrt{n}}^{T \sqrt{n}} e^{-i t x}\left[f^{n}\left(\frac{t}{\sqrt{n}}\right)-g_{5}^{n}\left(\frac{t}{\sqrt{n}}\right)\right] d t \\
& +\frac{1}{2 \pi} \int_{|t| \geqslant T \sqrt{n}} e^{-i t x} f^{n}\left(\frac{t}{\sqrt{n}}\right) d t \\
& -\frac{1}{2 \pi} \int_{|t| \geqslant T \sqrt{n}} e^{-i t x} g_{5}^{n}\left(\frac{t}{\sqrt{n}}\right) d t .
\end{aligned}
$$

Абсолютные величины двух последних слагаемых в правой части (2) допускают оценки

$$
\begin{aligned}
& \frac{1}{2 \pi} \int_{|t| \geqslant T \sqrt{n}}\left|f\left(\frac{t}{\sqrt{n}}\right)\right|^{n} d t \leqslant \frac{\sqrt{n}}{\pi} \alpha^{n-\nu}(T) \int_{T}^{\infty}|f(t)|^{\nu} d t=A_{n}(T), \\
& \left|\frac{1}{2 \pi} \int_{|t| \geqslant T \sqrt{n}} e^{-i t x} g_{5}^{n}\left(\frac{t}{\sqrt{n}}\right) d t\right| \leqslant \frac{1}{\pi} \int_{|t| \geqslant T \sqrt{n}} e^{-t^{2} / 2} d t=L_{0}(T \sqrt{n}) .
\end{aligned}
$$

Мы видим, что $A_{n}(T)$ и $L_{0}(T \sqrt{n})$ убывают экспоненциально быстро при росте $n$.

Для первого слагаемого в правой части равенства (2) сделаем следующее известное преобразование:

$$
\frac{1}{2 \pi} \int_{-T \sqrt{n}}^{T \sqrt{n}} e^{-i t x}\left[f^{n}\left(\frac{t}{\sqrt{n}}\right)-g_{5}^{n}\left(\frac{t}{\sqrt{n}}\right)\right] d t
$$




$$
\begin{aligned}
= & \frac{1}{2 \pi} \int_{-T \sqrt{n}}^{T \sqrt{n}} e^{-i t x} \sum_{j=0}^{n-1} f^{j}\left(\frac{t}{\sqrt{n}}\right) g_{5}^{n-j-1}\left(\frac{t}{\sqrt{n}}\right) e^{-t^{2} /(2 n)} \\
& \times\left[e^{t^{2} /(2 n)} f\left(\frac{t}{\sqrt{n}}\right)-e^{t^{2} /(2 n)} g_{5}\left(\frac{t}{\sqrt{n}}\right)\right] d t .
\end{aligned}
$$

Из разложения (1) при $m=4$ следует, что

$$
\begin{aligned}
e^{t^{2} /(2 n)} f\left(\frac{t}{\sqrt{n}}\right)= & \sum_{k=0}^{5} \theta_{k}\left(\frac{i t}{\sqrt{n}}\right)^{k}+\gamma e^{t^{2} /(2 n)}\left\|\theta_{6}\right\|\left(\frac{t}{\sqrt{n}}\right)^{6} \\
& +\gamma e^{t^{2} /(2 n)}\left\|\theta_{7}^{(5)}\right\|\left(\frac{t}{\sqrt{n}}\right)^{7}
\end{aligned}
$$

и почти очевидно, что

$$
\begin{aligned}
e^{t^{2} /(2 n)} g_{5}\left(\frac{t}{\sqrt{n}}\right)= & \exp \left\{\theta_{3}\left(\frac{i t}{\sqrt{n}}\right)^{3}+\theta_{5}\left(\frac{i t}{\sqrt{n}}\right)^{5}\right\}=\exp \left\{\theta_{3}\left(\frac{i t}{\sqrt{n}}\right)^{3}\right\} \\
& +\theta_{5}\left(\frac{i t}{\sqrt{n}}\right)^{5} \exp \left\{\theta_{3}\left(\frac{i t}{\sqrt{n}}\right)^{3}\right\}+\gamma \frac{\theta_{5}^{2}}{2}\left(\frac{i t}{\sqrt{n}}\right)^{10} \\
= & 1+\theta_{3}\left(\frac{i t}{\sqrt{n}}\right)^{3}+\gamma \frac{\theta_{3}^{2}}{2}\left(\frac{t}{\sqrt{n}}\right)^{6}+\theta_{5}\left(\frac{i t}{\sqrt{n}}\right)^{5} \\
& +\gamma \theta_{3} \theta_{5}\left(\frac{t}{\sqrt{n}}\right)^{8}+\gamma \frac{\theta_{5}^{2}}{2}\left(\frac{t}{\sqrt{n}}\right)^{10} .
\end{aligned}
$$

Из (4) и (5) следует, что разность в квадратных скобках в правой части (3) равна $\theta_{4}(i t / \sqrt{n})^{4}+r_{5}(t)$, где $r_{5}(t)$ есть сумма (с соответствующими знаками) тех слагаемых в правых частях (4) и (5), которые содержат функции $\gamma$. Поэтому правая часть (3) равна

$$
\frac{\theta_{4}}{2 \pi} \int_{-T \sqrt{n}}^{T \sqrt{n}}\left(\frac{i t}{\sqrt{n}}\right)^{4} e^{-i t x} \sum_{j=0}^{n-1} f^{j}\left(\frac{t}{\sqrt{n}}\right) g_{5}^{n-j-1}\left(\frac{t}{\sqrt{n}}\right) e^{-t^{2} /(2 n)} d t+R_{5}
$$

где

$$
\begin{aligned}
\left|R_{5}\right| & \leqslant \sum_{j=0}^{n-1} \frac{1}{2 \pi} \int_{-T \sqrt{n}}^{T \sqrt{n}}\left|e^{-i t x}\right|\left|f^{j}\left(\frac{t}{\sqrt{n}}\right)\right|\left|g_{5}^{n-j-1}\left(\frac{t}{\sqrt{n}}\right)\right|\left|e^{-t^{2} /(2 n)}\right|\left|r_{5}(t)\right| d t \\
& \leqslant \sum_{j=0}^{n-1} \frac{1}{2 \pi} \int_{-T \sqrt{n}}^{T \sqrt{n}} \mu^{n-1}\left(\frac{t}{\sqrt{n}}\right)\left|r_{5}(t)\right| d t \\
& \leqslant \frac{\left\|\theta_{6}\right\| B_{6, n-1}}{(\sqrt{n})^{4}}+\frac{\theta_{3}^{2}}{2} \frac{B_{6, n}}{(\sqrt{n})^{4}}+\frac{\left\|\theta_{7}^{(5)}\right\| B_{7, n-1}}{(\sqrt{n})^{5}}+\frac{\left|\theta_{3} \theta_{5}\right| B_{8, n}}{(\sqrt{n})^{6}}+\frac{\theta_{5}^{2}}{2} \frac{B_{10, n-1}}{(\sqrt{n})^{8}} .
\end{aligned}
$$

Все слагаемые в правой части последнего неравенства входят в оценку остаточной части разложения теоремы 1. 
Рассмотрим первое слагаемое в (6). Его можно записать в виде

$$
\begin{gathered}
\frac{\theta_{4}}{2 \pi n^{2}} \int_{-T \sqrt{n}}^{T \sqrt{n}}(i t)^{4} e^{-i t x} \sum_{j=1}^{n-1} g_{5}^{n-j-1}\left(\frac{t}{\sqrt{n}}\right)\left[f^{j}\left(\frac{t}{\sqrt{n}}\right)-g_{3}^{j}\left(\frac{t}{\sqrt{n}}\right)\right] e^{-t^{2} /(2 n)} d t \\
+\frac{\theta_{4}}{2 \pi n^{2}} \int_{-T \sqrt{n}}^{T \sqrt{n}}(i t)^{4} e^{-i t x} \sum_{j=0}^{n-1} g_{5}^{n-j-1}\left(\frac{t}{\sqrt{n}}\right) g_{3}^{j}\left(\frac{t}{\sqrt{n}}\right) g(t) d t .
\end{gathered}
$$

Второе слагаемое в (7) равно

$$
\begin{aligned}
& \frac{\theta_{4}}{2 \pi(\sqrt{n})^{4}} \int_{-T \sqrt{n}}^{T \sqrt{n}}(i t)^{4} \exp \left\{-i t x-\frac{t^{2}}{2}\right\} \\
& \times \exp \left\{\frac{n-1}{n} \theta_{3} \frac{(i t)^{3}}{\sqrt{n}}\right\} \sum_{j=0}^{n-1} \exp \left\{\frac{n-j-1}{n} \theta_{5} \frac{(i t)^{5}}{(\sqrt{n})^{3}}\right\} d t \\
& =\frac{\theta_{4}}{2 \pi(\sqrt{n})^{2}} \int_{-T \sqrt{n}}^{T \sqrt{n}}(i t)^{4} \exp \left\{-i t x-\frac{t^{2}}{2}\right\} \\
& \times \exp \left\{\frac{n-1}{n} \theta_{3} \frac{(i t)^{3}}{\sqrt{n}}\right\} d t+R_{6},
\end{aligned}
$$

где

$$
\begin{aligned}
\left|R_{6}\right| & \leqslant \frac{\left|\theta_{4}\right|}{2 \pi(\sqrt{n})^{4}} \int_{-T \sqrt{n}}^{T \sqrt{n}}|t|^{4} e^{-t^{2} / 2} \sum_{j=0}^{n-2} \frac{n-j-1}{n} \frac{\left|\theta_{5}\right||t|^{5}}{(\sqrt{n})^{3}} d t \\
& =\frac{\left|\theta_{4} \theta_{5}\right|(n-1)}{2 \pi \cdot 2(\sqrt{n})^{7}} \int_{-T \sqrt{n}}^{T \sqrt{n}}|t|^{9} e^{-t^{2} / 2} d t \leqslant \frac{\left|\theta_{4} \theta_{5}\right| B_{9}}{2(\sqrt{n})^{5}} .
\end{aligned}
$$

Последняя величина также входит в оценку остаточной части разложения теоремы 1. Первое слагаемое в правой части (8) равно

$$
\begin{gathered}
\frac{\theta_{4}}{2 \pi(\sqrt{n})^{2}} \int_{-T \sqrt{n}}^{T \sqrt{n}}(i t)^{4} \exp \left\{-i t x-\frac{t^{2}}{2}\right\} \exp \left\{\frac{n-1}{n} \theta_{3} \frac{(i t)^{3}}{\sqrt{n}}\right\} \\
=\frac{\theta_{4}}{2 \pi(\sqrt{n})^{2}} \int_{-T \sqrt{n}}^{T \sqrt{n}}(i t)^{4} \exp \left\{-i t x-\frac{t^{2}}{2}\right\} \\
\times\left(1+\frac{n-1}{n} \theta_{3} \frac{(i t)^{3}}{\sqrt{n}}+\gamma \frac{\theta_{3}^{2}}{2} \frac{t^{6}}{(\sqrt{n})^{2}}\right) d t \\
=\frac{\theta_{4}}{(\sqrt{n})^{2}} H_{4}(x) \varphi(x)+\frac{n-1}{n} \frac{\theta_{3} \theta_{4}}{(\sqrt{n})^{3}} H_{7}(x) \varphi(x)+R_{7},
\end{gathered}
$$

где

$$
\left|R_{7}\right| \leqslant \frac{\left|\theta_{4}\right| L_{4}(T \sqrt{n})}{(\sqrt{n})^{2}}+\frac{\left|\theta_{3} \theta_{4}\right| L_{7}(T \sqrt{n})}{(\sqrt{n})^{3}}+\frac{\theta_{3}^{2}\left|\theta_{4}\right|}{2} \frac{B_{10}}{(\sqrt{n})^{4}} .
$$

Все слагаемые в правой части последнего неравенства входят в оценку остаточной части разложения теоремы 1. Здесь мы воспользовались тем, что

$$
\frac{1}{2 \pi} \int_{-\infty}^{\infty}(i t)^{l} e^{-i t x-t^{2} / 2} d t=H_{l}(x) \varphi(x), \quad l=0,1,2, \ldots
$$


Рассмотрим первое слагаемое в (7). Применив преобразование, аналогичное тому, которое уже использовалось в равенстве (3), его можно записать в виде

$$
\begin{aligned}
& \frac{\theta_{4}}{2 \pi(\sqrt{n})^{4}} \int_{-T \sqrt{n}}^{T \sqrt{n}}(i t)^{4} e^{-i t x} \sum_{j=1}^{n-1} g_{5}^{n-j-1}\left(\frac{t}{\sqrt{n}}\right) e^{-t^{2} /(2 n)} \\
& \times\left[\sum_{j_{0}=0}^{j-1} f^{j_{0}}\left(\frac{t}{\sqrt{n}}\right) g_{3}^{j-j_{0}-1}\left(\frac{t}{\sqrt{n}}\right) e^{-t^{2} /(2 n)}\right. \\
& \left.\times\left\{e^{t^{2} /(2 n)} f\left(\frac{t}{\sqrt{n}}\right)-e^{t^{2} /(2 n)} g_{3}\left(\frac{t}{\sqrt{n}}\right)\right\}\right] d t .
\end{aligned}
$$

Нетрудно проверить, что величину, стоящую в фигурных скобках, можно записать в виде

$$
\gamma e^{t^{2} /(2 n)}\left\|\theta_{4}\right\|\left|\frac{t}{\sqrt{n}}\right|^{4}+\gamma e^{t^{2} /(2 n)}\left\|\theta_{5}^{(3)}\right\|\left|\frac{t}{\sqrt{n}}\right|^{5}+\frac{\gamma \theta_{3}^{2}}{2}\left|\frac{t}{\sqrt{n}}\right|^{6},
$$

поэтому абсолютная величина (9) не превосходит

$$
\begin{aligned}
& \frac{\left|\theta_{4}\right|}{2 \pi(\sqrt{n})^{4}} \int_{-T \sqrt{n}}^{T \sqrt{n}} t^{4} \mu^{n-1}\left(\frac{t}{\sqrt{n}}\right) \\
& \quad \times \sum_{j=1}^{n-1} \sum_{j_{0}=0}^{j-1}\left[\left\|\theta_{4}\right\|\left|\frac{t}{\sqrt{n}}\right|^{4}+\left\|\theta_{5}^{(3)}\right\|\left|\frac{t}{\sqrt{n}}\right|^{5}+\frac{\theta_{3}^{2}}{2}\left|\frac{t}{\sqrt{n}}\right|^{6} e^{-t^{2} /(2 n)}\right] d t \\
& =C_{n}^{2}\left[\frac{\left|\theta_{4}\right|\left\|\theta_{4}\right\| B_{8, n-1}}{(\sqrt{n})^{8}}+\frac{\left|\theta_{4}\right|\left\|\theta_{5}^{(3)}\right\| B_{9, n-1}}{(\sqrt{n})^{9}}+\frac{\theta_{3}^{2}\left|\theta_{4}\right| B_{10, n}}{2(\sqrt{n})^{10}}\right] \\
& \leqslant \frac{1}{2}\left[\frac{\left|\theta_{4}\right|\left\|\theta_{4}\right\| B_{4, n-1}}{(\sqrt{n})^{8}}+\frac{\left|\theta_{4}\right|\left\|\theta_{5}^{(3)}\right\| B_{5, n-1}}{(\sqrt{n})^{9}}+\frac{\theta_{3}^{2}\left|\theta_{4}\right| B_{6, n}}{2(\sqrt{n})^{10}}\right] .
\end{aligned}
$$

Слагаемые в правой части содержатся в оценке остаточной части разложения теоремы 1.

Таким образом,

$$
p_{n}(x)-q_{n}(x)=\frac{\theta_{4}}{n} H_{4}(x) \varphi(x)+\frac{\theta_{3} \theta_{4}}{(\sqrt{n})^{3}} H_{7}(x) \varphi(x)+R_{8},
$$

где абсолютная величина $R_{8}$ не превосходит суммы тех величин, входящих в оценку остаточной части разложения теоремы 1 , о которых говорилось выше.

Теперь для завершения доказательства этой теоремы нам достаточно написать разложение для $q_{n}(x)$ :

$$
\begin{aligned}
q_{n}(x) & =\frac{1}{2 \pi} \int_{-\infty}^{\infty} e^{-i t x} g_{5}^{n}\left(\frac{t}{\sqrt{n}}\right) d t \\
& =\frac{1}{2 \pi} \int_{-\infty}^{\infty} e^{-i t x-t^{2} / 2} \exp \left\{\theta_{3} \frac{(i t)^{3}}{\sqrt{n}}+\theta_{5} \frac{(i t)^{5}}{(\sqrt{n})^{3}}\right\} d t
\end{aligned}
$$




$$
\begin{aligned}
= & \frac{1}{2 \pi} \int_{-\infty}^{\infty} e^{-i t x-t^{2} / 2} \\
& \quad \times\left(1+\theta_{3} \frac{(i t)^{3}}{\sqrt{n}}+\frac{\theta_{3}^{2}}{2} \frac{(i t)^{6}}{(\sqrt{n})^{2}}+\frac{\theta_{3}^{3}}{6} \frac{(i t)^{9}}{(\sqrt{n})^{3}}+\gamma \frac{\theta_{3}^{4}}{4 !} \frac{(i t)^{12}}{(\sqrt{n})^{4}}\right) d t \\
& +\frac{1}{2 \pi} \int_{-\infty}^{\infty} e^{-i t x-t^{2} / 2}\left(\theta_{5} \frac{(i t)^{5}}{(\sqrt{n})^{3}}+\gamma \theta_{3} \theta_{5} \frac{(i t)^{8}}{(\sqrt{n})^{4}}+\gamma \frac{\theta_{5}^{2}}{2} \frac{(i t)^{10}}{(\sqrt{n})^{6}}\right) d t \\
= & \varphi(x)+\frac{\theta_{3}}{\sqrt{n}} H_{3}(x) \varphi(x)+\frac{\theta_{5}}{(\sqrt{n})^{3}} H_{5}(x) \varphi(x)+\frac{\theta_{3}^{2}}{2(\sqrt{n})^{2}} H_{6}(x) \varphi(x) \\
& +\frac{\theta_{3}^{3}}{6(\sqrt{n})^{3}} H_{9}(x) \varphi(x)+R_{9},
\end{aligned}
$$

где

$$
\left|R_{9}\right| \leqslant \frac{\theta_{3}^{4}}{4 !} \frac{B_{12}}{(\sqrt{n})^{4}}+\frac{\left|\theta_{3} \theta_{5}\right| B_{8}}{(\sqrt{n})^{4}}+\frac{\theta_{5}^{2}}{2} \frac{B_{10}}{(\sqrt{n})^{6}} .
$$

Слагаемые правой части этого неравенства входят в оценку остаточной части разложения теоремы 1 . Теорема 1 доказана.

3 а м е ч а н и е. Слагаемое главной части разложения теоремы 1 , связанное с многочленом $H_{7}(x)$, можно было разбить на два и одно из них, $\left(\theta_{3} \theta_{4} /(\sqrt{n})^{5}\right) H_{7}(x) \varphi(x)$, оставить в главной части разложения, а оценку второго $\left|-\left(\theta_{3} \theta_{4} /(\sqrt{n})^{5}\right) H_{7}(x) \varphi(x)\right| \leqslant\left|\theta_{3} \theta_{4}\right| B_{7} /(\sqrt{n})^{5}$ добавить в оценку остаточной части разложения. Так мы бы получили разложение Эджворта-Крамера, записанное в форме разложения Грама-Шарлье [1].

Д ок а з а т ль с т о те о ремы 2. Для того чтобы из доказательства теоремы 1 получить доказательство теоремы 2, необходимо сделать следующие изменения.

В правой части (4) сумму двух последних слагаемых следует заменить на $\theta_{6}(i t / \sqrt{n})^{6}+\gamma\left\|\theta_{7}\right\|(t / \sqrt{n})^{7}+\gamma\left\|\theta_{8}^{(6)}\right\|(t / \sqrt{n})^{8}$, а в правой части равенства (5) величину $\gamma\left(\theta_{3}^{2} / 2\right)(t / \sqrt{n})^{6}$ заменить на $\left(\theta_{3}^{2} / 2\right)(i t / \sqrt{n})^{6}+$ $\gamma\left(\theta_{3}^{3} / 3\right.$ ! $)(t / \sqrt{n})^{9}$. При этом в $(6)$ вместо величины $\theta_{4}(i t / \sqrt{n})^{4}$ будет участвовать величина $\theta_{4}(i t / \sqrt{n})^{4}+\left(\theta_{6}-\theta_{3}^{2} / 2\right)(i t / \sqrt{n})^{6}$, а для величины $R_{5}$ из $(6)$ будет справедливо неравенство

$$
\left|R_{5}\right| \leqslant \frac{\left\|\theta_{7}\right\| B_{7, n-1}}{(\sqrt{n})^{5}}+\frac{\left\|\theta_{8}^{(6)}\right\| B_{8, n-1}}{(\sqrt{n})^{6}}+\frac{\left|\theta_{3} \theta_{5}\right| B_{8, n}}{(\sqrt{n})^{6}}+\frac{\left|\theta_{3}^{3}\right|}{3 !} \frac{B_{9, n}}{(\sqrt{n})^{7}}+\frac{\theta_{5}^{2}}{2} \frac{B_{10, n-1}}{(\sqrt{n})^{8}} .
$$

Аналог (7) следует записать в виде

$$
\begin{aligned}
& \frac{\theta_{4}}{2 \pi(\sqrt{n})^{4}} \int_{-T \sqrt{n}}^{T \sqrt{n}}(i t)^{4} e^{-i t x} \\
& \quad \times \sum_{j=1}^{n-1} g_{5}^{n-j-1}\left(\frac{t}{\sqrt{n}}\right)\left[f^{j}\left(\frac{t}{\sqrt{n}}\right)-g_{3}^{j}\left(\frac{t}{\sqrt{n}}\right)\right] e^{-t^{2} /(2 n)} d t \\
& +\frac{\theta_{4}}{2 \pi(\sqrt{n})^{4}} \int_{-T \sqrt{n}}^{T \sqrt{n}}(i t)^{4} e^{-i t x} \sum_{j=0}^{n-1} g_{5}^{n-j-1}\left(\frac{t}{\sqrt{n}}\right) g_{3}^{j}\left(\frac{t}{\sqrt{n}}\right) e^{-t^{2} /(2 n)} d t
\end{aligned}
$$




$$
\begin{aligned}
&+\frac{\left(\theta_{6}-\theta_{3}^{2} / 2\right)}{2 \pi(\sqrt{n})^{6}} \int_{-T \sqrt{n}}^{T \sqrt{n}}(i t)^{6} e^{-i t x} \\
& \times \sum_{j=1}^{n-1} g_{5}^{n-j-1}\left(\frac{t}{\sqrt{n}}\right)\left[f^{j}\left(\frac{t}{\sqrt{n}}\right)-g_{3}^{j}\left(\frac{t}{\sqrt{n}}\right)\right] e^{-t^{2} /(2 n)} d t \\
&+\frac{\left(\theta_{6}-\theta_{3}^{2} / 2\right)}{2 \pi(\sqrt{n})^{6}} \int_{-T \sqrt{n}}^{T \sqrt{n}}(i t)^{6} e^{-i t x} \sum_{j=0}^{n-1} g_{5}^{n-j-1}\left(\frac{t}{\sqrt{n}}\right) g_{3}^{j}\left(\frac{t}{\sqrt{n}}\right) e^{-t^{2} /(2 n)} d t .
\end{aligned}
$$

Последнее слагаемое в (10) равно

$$
\begin{gathered}
\frac{\left(\theta_{6}-\theta_{3}^{2} / 2\right)}{2 \pi(\sqrt{n})^{6}} \int_{-T \sqrt{n}}^{T \sqrt{n}}(i t)^{6} \exp \left\{-i t x-\frac{t^{2}}{2}\right\} \exp \left\{\frac{n-1}{n} \theta_{3} \frac{(i t)^{3}}{\sqrt{n}}\right\} \\
\times \sum_{j=0}^{n-1} \exp \left\{\frac{j}{n} \theta_{5} \frac{(i t)^{5}}{(\sqrt{n})^{3}}\right\} d t \\
=\frac{\left(\theta_{6}-\theta_{3}^{2} / 2\right)}{2 \pi(\sqrt{n})^{4}} \int_{-T \sqrt{n}}^{T \sqrt{n}}(i t)^{6} \exp \left\{-i t x-\frac{t^{2}}{2}\right\} \\
\times \exp \left\{\frac{n-1}{n} \theta_{3} \frac{(i t)^{3}}{\sqrt{n}}\right\} d t+R_{10}
\end{gathered}
$$

где

$$
\left|R_{10}\right| \leqslant \frac{\left|\theta_{6}-\theta_{3}^{2} / 2\right|}{2 \pi(\sqrt{n})^{6}} \int_{-T \sqrt{n}}^{T \sqrt{n}}|t|^{6} e^{-t^{2} / 2} \sum_{j=1}^{n-2} \frac{j}{n} \frac{\left|\theta_{5}\right||t|^{5}}{(\sqrt{n})^{3}} d t \leqslant\left|\theta_{6}-\frac{\theta_{3}^{2}}{2}\right|\left|\theta_{5}\right| \frac{B_{11}}{2(\sqrt{n})^{7}} .
$$

Первое слагаемое в правой части (11) равно

$$
\frac{\left(\theta_{6}-\theta_{3}^{2} / 2\right)}{2 \pi(\sqrt{n})^{4}} \int_{-\infty}^{\infty}(i t)^{6} e^{-i t x-t^{2} / 2} d t+R_{11}=\frac{\left(\theta_{6}-\theta_{3}^{2} / 2\right)}{(\sqrt{n})^{4}} H_{6}(x) \varphi(x)+R_{11}
$$

где

$$
\begin{aligned}
\left|R_{11}\right| & \leqslant\left|\theta_{6}-\frac{\theta_{3}^{2}}{2}\right| \frac{1}{2 \pi(\sqrt{n})^{4}} \int_{-\infty}^{\infty} t^{6} e^{-t^{2} / 2}\left|\theta_{3}\right| \frac{|t|^{3}}{\sqrt{n}} d t+\left|\theta_{6}-\frac{\theta_{3}^{2}}{2}\right| \frac{L_{6}(T \sqrt{n})}{(\sqrt{n})^{4}} \\
& \leqslant\left|\theta_{6}-\frac{\theta_{3}^{2}}{2}\right| \frac{\left|\theta_{3}\right| B_{9}}{(\sqrt{n})^{5}}+\left|\theta_{6}-\frac{\theta_{3}^{2}}{2}\right| \frac{L_{6}(T \sqrt{n})}{(\sqrt{n})^{4}} .
\end{aligned}
$$

Второе слагаемое в (10), как и при доказательстве теоремы 1 (см. равенство (8)), может быть представлено в виде

$$
\frac{\theta_{4}}{2 \pi(\sqrt{n})^{2}} \int_{-T \sqrt{n}}^{T \sqrt{n}}(i t)^{4} \exp \left\{-i t x-\frac{t^{2}}{2}\right\} \exp \left\{\frac{n-1}{n} \theta_{3} \frac{(i t)^{3}}{\sqrt{n}}\right\} d t+R_{6}
$$

где $\left|R_{6}\right| \leqslant\left|\theta_{4} \theta_{5}\right| B_{9} /\left[2(\sqrt{n})^{5}\right]$. Эта оценка устанавливается точно так же, как при доказательстве теоремы 1 . Первое слагаемое в (12) рассматривается с помощью чуть более точного разложения экспоненты, 
содержащей $\theta_{3}$, чем в доказательстве теоремы 1 . Именно, эта величина равна

$$
\begin{aligned}
& \frac{\theta_{4}}{2 \pi(\sqrt{n})^{2}} \int_{-T \sqrt{n}}^{T \sqrt{n}}(i t)^{4} \exp \left\{-i t x-\frac{t^{2}}{2}\right\} \\
& \quad \times\left(1+\frac{n-1}{n} \theta_{3} \frac{(i t)^{3}}{\sqrt{n}}+\left(\frac{n-1}{n}\right)^{2} \frac{\theta_{3}^{2}}{2} \frac{t^{6}}{(\sqrt{n})^{2}}+\gamma \frac{\theta_{3}^{3}}{3 !} \frac{t^{9}}{(\sqrt{n})^{3}}\right) d t \\
& =\frac{\theta_{4}}{(\sqrt{n})^{2}} H_{4}(x) \varphi(x)+\frac{n-1}{n} \frac{\theta_{3} \theta_{4}}{(\sqrt{n})^{3}} H_{7}(x) \varphi(x) \\
& \quad+\left(\frac{n-1}{n}\right)^{2} \frac{\theta_{3}^{2} \theta_{4}}{2(\sqrt{n})^{4}} H_{10}(x) \varphi(x)+R_{12},
\end{aligned}
$$

где

$$
\left|R_{12}\right| \leqslant \frac{\left|\theta_{4}\right| L_{4}(T \sqrt{n})}{(\sqrt{n})^{2}}+\frac{\left|\theta_{3} \theta_{4}\right| L_{7}(T \sqrt{n})}{(\sqrt{n})^{3}}+\frac{\left|\theta_{3}^{2} \theta_{4}\right| L_{10}(T \sqrt{n})}{2(\sqrt{n})^{4}}+\frac{\left|\theta_{3}^{3} \theta_{4}\right|}{2} \frac{B_{13}}{(\sqrt{n})^{5}} .
$$

Третье слагаемое в (10) рассматривается аналогично тому, как мы рассматривали первое слагаемое в (7), и для его абсолютной величины получается оценка

$$
\frac{\left|\theta_{6}-\theta_{3}^{2} / 2\right|\left\|\theta_{4}\right\| B_{10, n-1}}{2(\sqrt{n})^{6}}+\frac{\left|\theta_{6}-\theta_{3}^{2} / 2\right|\left\|\theta_{5}^{(3)}\right\| B_{11, n-1}}{(\sqrt{n})^{7}}+\frac{\left|\theta_{6}-\theta_{3}^{2} / 2\right| \theta_{3}^{2} B_{12, n}}{2(\sqrt{n})^{8}}
$$

Рассмотрим первое слагаемое в (10). Оно совпадает с первым слагаемым в (7), для которого в доказательстве теоремы 2 была получена формула (9). Но для преобразования первого слагаемого из (10) нам придется использовать более тонкие соображения, чем при доказательстве теоремы 1. Заметим, что

$$
\begin{aligned}
e^{t^{2} /(2 n)} f\left(\frac{t}{\sqrt{n}}\right)= & \sum_{k=0}^{4} \theta_{k}\left(\frac{i t}{\sqrt{n}}\right)^{k}+\gamma e^{t^{2} /(2 n)}\left\|\theta_{5}\right\|\left(\frac{t}{\sqrt{n}}\right)^{5} \\
& +\gamma e^{t^{2} /(2 n)}\left\|\theta_{6}^{(4)}\right\|\left(\frac{t}{\sqrt{n}}\right)^{6}, \\
e^{t^{2} /(2 n)} g_{3}\left(\frac{t}{\sqrt{n}}\right)= & \exp \left\{\theta_{3}\left(\frac{i t}{\sqrt{n}}\right)^{3}\right\}=1+\theta_{3}\left(\frac{i t}{\sqrt{n}}\right)^{3}+\gamma \frac{\theta_{3}^{2}}{2}\left(\frac{t}{\sqrt{n}}\right)^{6} .
\end{aligned}
$$

Поэтому для рассматриваемого слагаемого величина (9) равна

$$
\begin{aligned}
& \frac{\theta_{4}}{2 \pi(\sqrt{n})^{4}} \int_{-T \sqrt{n}}^{T \sqrt{n}}(i t)^{4} e^{-i t x} \sum_{j=1}^{n-1} \sum_{j_{0}=0}^{j-1} f^{j_{0}}\left(\frac{t}{\sqrt{n}}\right) \\
& \quad \times e^{-t^{2} /(2 n)} g_{3}^{j-j_{0}-1}\left(\frac{t}{\sqrt{n}}\right) e^{-t^{2} /(2 n)} g_{5}^{n-j-1}\left(\frac{t}{\sqrt{n}}\right) \theta_{4}\left(\frac{i t}{\sqrt{n}}\right)^{4} d t+R_{13} \\
& =\frac{\theta_{4}^{2}}{2 \pi(\sqrt{n})^{8}} \int_{-T \sqrt{n}}^{T \sqrt{n}}(i t)^{8} e^{-i t x} \sum_{j=1}^{n-1} \sum_{j_{0}=0}^{j-1} f^{j_{0}}\left(\frac{t}{\sqrt{n}}\right) \\
& \quad \times e^{-t^{2} /(2 n)} g_{3}^{j-j_{0}-1}\left(\frac{t}{\sqrt{n}}\right) e^{-t^{2} /(2 n)} g_{5}^{n-j-1}\left(\frac{t}{\sqrt{n}}\right) d t+R_{13},
\end{aligned}
$$


где

$$
\begin{aligned}
\left|R_{13}\right| \leqslant \sum_{j=1}^{n-1} \sum_{j=0}^{j-1} \frac{\left|\theta_{4}\right|}{2 \pi(\sqrt{n})^{4}} \int_{-T \sqrt{n}}^{T \sqrt{n}} t^{4} \mu^{n-1}\left(\frac{t}{\sqrt{n}}\right) \\
\times\left[\left\|\theta_{5}\right\|\left|\frac{t}{\sqrt{n}}\right|^{5}+\left\|\theta_{6}^{(4)}\right\|\left|\frac{t}{\sqrt{n}}\right|^{6}+\frac{\theta_{3}^{2}}{2}\left|\frac{t}{\sqrt{n}}\right|^{6} e^{\left.-t^{2} /(2 n)\right] d t}\right. \\
\leqslant \frac{\left|\theta_{4}\right|\left\|\theta_{5}\right\| B_{9, n-1}}{2(\sqrt{n})^{5}}+\frac{\left|\theta_{4}\right|\left\|\theta_{6}^{(4)}\right\| B_{10, n-1}}{2(\sqrt{n})^{6}}+\frac{\theta_{3}^{2}\left|\theta_{4}\right| B_{10, n}}{4(\sqrt{n})^{6}}
\end{aligned}
$$

Первое слагаемое в правой части (13) равно

$$
\begin{aligned}
& \frac{\theta_{4}^{2}}{2 \pi(\sqrt{n})^{8}} \sum_{j=1}^{n-1} \sum_{j_{0}=0}^{j-1} \int_{-T \sqrt{n}}^{T \sqrt{n}}(i t)^{8} e^{-i t x} f^{j_{0}}\left(\frac{t}{\sqrt{n}}\right) \\
& \quad \times e^{-t^{2} /(2 n)} g_{3}^{j-j_{0}-1}\left(\frac{t}{\sqrt{n}}\right) e^{-t^{2} /(2 n)} g_{5}^{n-j-1}\left(\frac{t}{\sqrt{n}}\right) d t \\
& =\frac{\theta_{4}^{2}}{2 \pi(\sqrt{n})^{8}} \sum_{j=1}^{n-1} \sum_{j_{0}=0}^{j-1} \int_{-T \sqrt{n}}^{T \sqrt{n}}(i t)^{8} e^{-i t x} f^{j_{0}}\left(\frac{t}{\sqrt{n}}\right) \\
& \times \exp \left\{-\left(n-j_{0}\right) \frac{t^{2}}{2 n}+\left(n-j_{0}-2\right) \theta_{3} \frac{(i t)^{3}}{(\sqrt{n})^{3}}\right\} \\
& =\frac{\theta_{4}^{2}}{2 \pi(\sqrt{n})^{8}} \sum_{j_{0}=0}^{n-2} C_{n-1-j_{0}}^{1} \times \exp \left\{(n-j-1) \theta_{5} \frac{(i t)^{5}}{(\sqrt{n})^{5}}\right\} d t \\
& \quad \times \int_{-T \sqrt{n}}^{T \sqrt{n}}(i t)^{8} e^{-i t x} f^{j_{0}}\left(\frac{t}{\sqrt{n}}\right) \\
& \quad \times \exp \left\{-\left(n-j_{0}\right) \frac{t^{2}}{2 n}+\left(n-j_{0}-2\right) \theta_{3} \frac{(i t)^{3}}{(\sqrt{n})^{3}}\right\} d t+R_{14},(1)
\end{aligned}
$$

где

$$
\left|R_{14}\right| \leqslant \frac{\theta_{4}^{2}}{2 \pi(\sqrt{n})^{8}} \int_{-T \sqrt{n}}^{T \sqrt{n}}|t|^{8} e^{-t^{2} / 2} C_{n}^{3} \frac{\left|\theta_{5}\right||t|^{5}}{(\sqrt{n})^{5}} d t \leqslant \frac{\theta_{4}^{2}\left|\theta_{5}\right| B_{13}}{3 !(\sqrt{n})^{7}} .
$$

Первое слагаемое в правой части (14) равно

$$
\begin{aligned}
& \frac{\theta_{4}^{2}}{2 \pi(\sqrt{n})^{8}} \sum_{j_{0}=0}^{n-2} C_{n-1-j_{0}}^{1} \int_{-T \sqrt{n}}^{T \sqrt{n}}(i t)^{8} \exp \left\{-i t x-\frac{t^{2}}{2}\right\} f^{j_{0}}\left(\frac{t}{\sqrt{n}}\right) \\
& \times \exp \left\{j_{0} \frac{t^{2}}{2 n}+\left(n-j_{0}-2\right) \theta_{3} \frac{(i t)^{3}}{(\sqrt{n})^{3}}\right\} d t \\
& =\frac{\theta_{4}^{2}}{2 \pi(\sqrt{n})^{8}} \int_{-T \sqrt{n}}^{T \sqrt{n}}(i t)^{8} \exp \left\{-i t x-\frac{t^{2}}{2}\right\}
\end{aligned}
$$




$$
\begin{aligned}
& \times\left(\sum_{j_{0}=0}^{n-2} C_{n-1-j_{0}}^{1}\left[f^{j_{0}}\left(\frac{t}{\sqrt{n}}\right)-g_{3}^{j_{0}}\left(\frac{t}{\sqrt{n}}\right)\right]\right) \\
& \times \exp \left\{j_{0} \frac{t^{2}}{2 n}+\left(n-j_{0}-2\right) \theta_{3} \frac{(i t)^{3}}{(\sqrt{n})^{3}}\right\} d t \\
+\frac{\theta_{4}^{2}}{2 \pi(\sqrt{n})^{8}} \int_{-}^{T \sqrt{n}}(i t)^{8} \exp \left\{-i t x-\frac{t^{2}}{2}\right\} & \sum_{j_{0}=1}^{n-2} C_{n-1-j_{0}}^{1} \exp \left\{-j_{0} \frac{t^{2}}{2 n}+j_{0} \theta_{3}\left(\frac{i t}{\sqrt{n}}\right)^{3}\right\} \\
& \times \exp \left\{j_{0} \frac{t^{2}}{2 n}+\left(n-j_{0}-2\right) \theta_{3} \frac{(i t)^{3}}{(\sqrt{n})^{3}}\right\} d t .
\end{aligned}
$$

Второе слагаемое в правой части (15) равно

$$
\begin{aligned}
& \frac{\theta_{4}^{2}}{2 \pi(\sqrt{n})^{8}} \int_{-T \sqrt{n}}^{T \sqrt{n}}(i t)^{8} \exp \left\{-i t x-\frac{t^{2}}{2}\right\} \\
& \times \sum_{j_{0}=1}^{n-2} C_{n-1-j_{0}}^{1} \exp \left\{(n-2) \theta_{3} \frac{(i t)^{3}}{(\sqrt{n})^{3}}\right\} d t \\
& =\frac{\theta_{4}^{2} C_{n}^{2}}{2 \pi(\sqrt{n})^{8}} \int_{-T \sqrt{n}}^{T \sqrt{n}}(i t)^{8} \exp \left\{-i t x-\frac{t^{2}}{2}\right\} \exp \left\{(n-2) \theta_{3} \frac{(i t)^{3}}{(\sqrt{n})^{3}}\right\} d t \\
& =\frac{\theta_{4}^{2} C_{n}^{2} L_{8}(T \sqrt{n})}{(\sqrt{n})^{8}}+\frac{\theta_{4}^{2} C_{n}^{2}}{2 \pi(\sqrt{n})^{8}} \int_{-\infty}^{\infty}(i t)^{8} \exp \left\{-i t x-\frac{t^{2}}{2}\right\} d t \\
& \quad+\gamma \frac{\theta_{3} \theta_{4}^{2} C_{n}^{2}(n-2)}{2 \pi(\sqrt{n})^{11}} \int_{-T \sqrt{n}}^{T \sqrt{n}}(i t)^{11} \exp \left\{-i t x-\frac{t^{2}}{2}\right\} d t \\
& =\frac{\theta_{4}^{2} C_{n}^{2}}{2 \pi(\sqrt{n})^{8}} \int_{-\infty}^{\infty}(i t)^{8} \exp \left\{-i t x-\frac{t^{2}}{2}\right\} d t+R_{17} \\
& =\frac{\theta_{4}^{2} C_{n}^{2}}{(\sqrt{n})^{8} H_{8}(x) \varphi(x)+R_{17},}
\end{aligned}
$$

где

$$
\begin{aligned}
\left|R_{17}\right| & \leqslant \frac{\left|\theta_{3}\right| \theta_{4}^{2}}{4 \pi(\sqrt{n})^{5}} \int_{-T \sqrt{n}}^{T \sqrt{n}}(i t)^{11} e^{-t^{2} / 2} d t+\frac{\theta_{4}^{2} L_{8}(T \sqrt{n})}{2(\sqrt{n})^{4}} \\
& =\frac{\left|\theta_{3}\right| \theta_{4}^{2} B_{11}}{2(\sqrt{n})^{5}}+\frac{\theta_{4}^{2} L_{8}(T \sqrt{n})}{2(\sqrt{n})^{4}} .
\end{aligned}
$$

Первое слагаемое в правой части (15) равно

$$
\begin{aligned}
\frac{\theta_{4}^{2}}{2 \pi(\sqrt{n})^{8}} \sum_{j_{0}=1}^{n-2} C_{n-1-j_{0}}^{1} \int_{-T \sqrt{n}}^{T \sqrt{n}}(i t)^{8} \exp \left\{-i t x-\frac{t^{2}}{2}\right\} \\
\quad \times \exp \left\{j_{0} \frac{t^{2}}{2 n}+\left(n-j_{0}-2\right) \theta_{3} \frac{(i t)^{3}}{(\sqrt{n})^{3}}\right\}
\end{aligned}
$$




$$
\begin{aligned}
& \times\left[f^{j_{0}}\left(\frac{t}{\sqrt{n}}\right)-g_{3}^{j_{0}}\left(\frac{t}{\sqrt{n}}\right)\right] d t \\
& =\frac{\theta_{4}^{2}}{2 \pi(\sqrt{n})^{8}} \sum_{j_{0}=1}^{n-2} C_{n-1-j_{0}}^{1} \int_{-T \sqrt{n}}^{T \sqrt{n}}(i t)^{8} \exp \left\{-i t x-\frac{t^{2}}{2}\right\} \\
& \times \exp \left\{j_{0} \frac{t^{2}}{2 n}+\left(n-j_{0}-2\right) \theta_{3} \frac{(i t)^{3}}{(\sqrt{n})^{3}}\right\} \\
& \times\left[\sum_{j=1}^{j_{0}-1} f^{j}\left(\frac{t}{\sqrt{n}}\right) g_{3}^{j_{0}-j-1}\left(\frac{t}{\sqrt{n}}\right) e^{-t^{2} /(2 n)}\right. \\
& \left.\times\left\{e^{t^{2} /(2 n)} f\left(\frac{t}{\sqrt{n}}\right)-e^{t^{2} /(2 n)} g_{3}\left(\frac{t}{\sqrt{n}}\right)\right\}\right] d t \\
& =\frac{\theta_{4}^{2}}{2 \pi(\sqrt{n})^{8}} \sum_{j=0}^{n-3} C_{n-1-j}^{2} \int_{-T \sqrt{n}}^{T \sqrt{n}}(i t)^{8} \exp \left\{-i t x-\frac{t^{2}}{2}\right\} f^{j}\left(\frac{t}{\sqrt{n}}\right) \\
& \times \exp \left\{\frac{j t^{2}}{2 n}+(n-j-3) \theta_{3} \frac{(i t)^{3}}{(\sqrt{n})^{3}}\right\} \\
& \times\left\{e^{t^{2} /(2 n)} f\left(\frac{t}{\sqrt{n}}\right)-e^{t^{2} /(2 n)} g_{3}\left(\frac{t}{\sqrt{n}}\right)\right\} d t .
\end{aligned}
$$

Последнее выражение по абсолютной величине не превосходит

$$
\begin{aligned}
& \mid \frac{\theta_{4}^{2}(\sqrt{n})^{6}}{2 \pi 6(\sqrt{n})^{8}} \int_{-T \sqrt{n}}^{T \sqrt{n}} t^{8} \mu^{n-3}\left(\frac{t}{\sqrt{n}}\right) \\
& \quad \times\left(\gamma e^{t^{2} /(2 n)}\left\|\theta_{4}\right\|\left|\frac{t}{\sqrt{n}}\right|^{4}+\gamma e^{t^{2} /(2 n)}\left\|\theta_{5}^{(3)}\right\|\left|\frac{t}{\sqrt{n}}\right|^{5}+\gamma \frac{\theta_{3}^{2}}{2}\left|\frac{t}{\sqrt{n}}\right|^{6}\right) \mid d t \\
& \leqslant \frac{\left\|\theta_{4}\right\| \theta_{4}^{2} B_{12, n-4}}{6(\sqrt{n})^{6}}+\frac{\left\|\theta_{5}^{(3)}\right\| \theta_{4}^{2} B_{13, n-4}}{6(\sqrt{n})^{7}}+\frac{\theta_{3}^{2} \theta_{4}^{2} B_{14, n-3}}{12(\sqrt{n})^{8}} .
\end{aligned}
$$

Для завершения доказательства теоремы 2 нам достаточно написать разложение для $q_{n}(x)$, более точное, чем то, что использовалось ранее:

$$
\begin{aligned}
q_{n}(x)= & \frac{1}{2 \pi} \int_{-\infty}^{\infty} e^{-i t x} g_{5}^{n}\left(\frac{t}{\sqrt{n}}\right) d t \\
= & \frac{1}{2 \pi} \int_{-\infty}^{\infty} \exp \left\{-i t x-\frac{t^{2}}{2}\right\} \exp \left\{\theta_{3} \frac{(i t)^{3}}{\sqrt{n}}+\theta_{5} \frac{(i t)^{5}}{(\sqrt{n})^{3}}\right\} d t \\
= & \frac{1}{2 \pi} \int_{-\infty}^{\infty} \exp \left\{-i t x-\frac{t^{2}}{2}\right\}\left(1+\theta_{3} \frac{(i t)^{3}}{\sqrt{n}}+\frac{\theta_{3}^{2}}{2} \frac{(i t)^{6}}{(\sqrt{n})^{2}}+\frac{\theta_{3}^{3}}{6} \frac{(i t)^{9}}{(\sqrt{n})^{3}}\right. \\
& \left.\quad+\frac{\theta_{3}^{4}}{4 !} \frac{(i t)^{12}}{(\sqrt{n})^{4}}+\gamma \frac{\theta_{3}^{5}}{5 !} \frac{(i t)^{15}}{(\sqrt{n})^{5}}\right) d t \\
& \quad+\frac{1}{2 \pi} \int_{-\infty}^{\infty} \exp \left\{-i t x-\frac{t^{2}}{2}\right\}\left(\theta_{5} \frac{(i t)^{5}}{(\sqrt{n})^{3}}+\theta_{3} \theta_{5} \frac{(i t)^{8}}{(\sqrt{n})^{4}}\right. \\
& \left.+\gamma \frac{\theta_{3}^{2}}{2} \theta_{5} \frac{(i t)^{11}}{(\sqrt{n})^{5}}+\gamma \frac{\theta_{5}^{2}}{2} \frac{(i t)^{10}}{(\sqrt{n})^{6}}\right) d t
\end{aligned}
$$




$$
\begin{aligned}
= & \varphi(x)+\frac{\theta_{3}}{\sqrt{n}} H_{3}(x) \varphi(x)+\frac{\theta_{5}}{(\sqrt{n})^{3}} H_{5}(x) \varphi(x) \\
& +\frac{\theta_{3}^{2}}{2(\sqrt{n})^{2}} H_{6}(x) \varphi(x)+\frac{\theta_{3}^{3}}{6(\sqrt{n})^{3}} H_{9}(x) \varphi(x) \\
& +\frac{\theta_{3}^{4}}{4 !(\sqrt{n})^{4}} H_{12}(x) \varphi(x)+\frac{\theta_{3} \theta_{5}}{(\sqrt{n})^{4}} H_{8}(x) \varphi(x)+R_{18}
\end{aligned}
$$

где

$$
\left|R_{18}\right| \leqslant \frac{\theta_{3}^{5}}{5 !} \frac{B_{15}}{(\sqrt{n})^{5}}+\frac{\theta_{3}^{2}\left|\theta_{5}\right| B_{11}}{2(\sqrt{n})^{5}}+\frac{\theta_{5}^{2}}{2} \frac{B_{10}}{(\sqrt{n})^{6}} .
$$

Слагаемые правой части этого неравенства входят в оценку остаточной части разложения теоремы. Теорема 2 доказана.

Доказательство теоремы 3 проводится по той же схеме, что и доказательства теорем 1 и 2, при этом следует использовать заряд с характеристической функцией $g_{7}(t)$. Новых идей при этом не требуется, и поэтому доказательство теоремы 3 мы опускаем полностью.

\section{СПИСОК ЛИТЕРАТУРЫ}

1. Сенатов В. В. Об асимптотических разложениях в центральной предельной теореме с явными оценками остаточных членов. - Теория вероятн. и ее примен., 2006 , т. 51 , в. 4 , с. $810-816$. 